Check for updates

Cite this: Soft Matter, 2019, 15, 1676

Received 15th September 2018, Accepted 8th December 2018

DOI: $10.1039 / \mathrm{c} 8 \mathrm{sm} 01892 \mathrm{~h}$

rsc.li/soft-matter-journal

\section{Directed tubule growth from giant unilamellar vesicles in a thermal gradient $\dagger$}

\author{
Emma L. Talbot, Jurij Kotar, Lorenzo Di Michele (D) and Pietro Cicuta (D) *
}

\section{Introduction}

Living cells generate many shape changes in order to achieve their function, including changes in local membrane curvature, growth of lipid protuberances ${ }^{1}$ and compartments of complex shape. ${ }^{2}$ For example, lipid tubules are prominently featured in the Golgi apparatus ${ }^{3}$ and the endoplasmic reticulum, ${ }^{4}$ both as transport conduits ${ }^{3}$ and intermediaries for communication and material transfer between cells via vesicle formation. ${ }^{5}$ Controlled growth of protuberances from a membrane is related to membrane tension, ${ }^{6}$ and it is integral for cell mimicry and the transfer of lipids and proteins between cells. For example, vesicles budded from a membrane can be used for transport internal or external to the cell, ${ }^{7,8}$ while membrane curvature plays a key role in cell division. ${ }^{9}$

Protuberances from a membrane can be highly curved, enabling the sorting of lipids. For example, regions of higher curvature in tubules pulled from aspirated vesicles were found to be enriched in the liquid-disordered phase. ${ }^{10}$ The curvature preference of some lipid phases over others, based on differences in bending modulii, also presents a method for curvatureinduced domain patterning. ${ }^{11}$ Indeed, some proteins induce (or stabilise) curvature themselves, e.g. BAR (Bin, Amphiphysin, Rvs) domains,${ }^{12-14}$ enabling lipid selectivity. Such proteins (or protein networks) may work via scaffolding - offering a curved surface for

Department of Physics, Cavendish Laboratory, University of Cambridge,

JJ Thomson Avenue, Cambridge, CB3 OHE, UK. E-mail: pc245@cam.ac.uk;

Tel: +44 (0)1223 337462

$\dagger$ Electronic supplementary information (ESI) available. See DOI: 10.1039/c8sm01892h interaction with the lipid membrane, or by directly inserting into part of the membrane (e.g. the top half of a bilayer) and acting as a wedge that locally deforms the membrane. ${ }^{12}$ For example, the lateral separation of curvature-inducing membrane proteins mediates endocytosis. ${ }^{15}$ Conversely, the selection of lipids in highly curved tubules offers the potential to expel known composition daughter vesicles from a membrane, or to deliver other moieties that prefer the tube phase, e.g. surfactants/proteins.

Giant unilamellar vesicles (GUVs) share various properties with biological cell membranes whilst reducing the complexity significantly, and they provide an ideal chassis for "artificial cells", enabling compartmentalisation of functional material within a surrounding bilayer membrane. ${ }^{17}$ Vesicle networks with compartmentalisation are capable of step-wise synthetic chemistry reactions, ${ }^{18}$ mimicking organelles or functioning towards artificial tissue. These nano-reactors can trigger the release of chemicals/ cargo when exposed to external stimuli. ${ }^{19}$ Directed growth of membrane extensions is therefore a relevant feature with potential to advance synthetic biology. GUVs with a ternary composition of a saturated lipid, an unsaturated lipid, and a sterol can form domains of coexisting liquid phases (liquid-ordered, $\mathrm{L}_{\mathrm{o}}$, and liquid-disordered, $\mathrm{L}_{\mathrm{d}}$ ) below a mixing transition temperature, $T_{\mathrm{t}}{ }^{20-22}$ The most common morphology, minimising the line tension, is circular domains. The phase forming domains are the minority phase and thus depend on the composition of the vesicle, and on the phase diagram (particularly the tie lines of the mixture). ${ }^{23}$ For example, at a higher concentration of saturated lipid, domains will be of the liquid-ordered phase. ${ }^{24}$

Directed growth of tubules via a non-invasive method can provide the first stage towards developing interconnected vesicle 
compartments with "communication" for creating artificial tissue. Alternatively, directed growth of tubules as transport intermediaries would allow the expulsion of daughter vesicles towards hot/cold for thermally targeted delivery. Current methods for tubule growth from GUVs are either invasive (micro-manipulation, ${ }^{25}$ optical tweezing of a bead attached to the membrane ${ }^{26,27}$ and hydrodynamic flows on immobilized vesicles ${ }^{28}$ ) or do not offer control over the direction of tubule growth (motor-proteins, ${ }^{29}$ curvature-inducing proteins, ${ }^{12,30}$ and inclusion of wedge-shaped lipids ${ }^{31}$ ). So far, only confinement methods, ${ }^{32}$ aqueous phase separation, ${ }^{33,34}$ and chemically-triggered ejection ${ }^{35}$ offer a route to spontaneously grow tubules in a defined direction, but the second needs a specific interior solution, and the latter requires local injection of a chemical.

In this paper, we explore the non-equilibrium conditions induced by thermal gradients as an unobtrusive method for directed tubule growth in GUVs towards hot or cold. This thermal method is of relevence to the intracellular environment, as the temperature difference between organelles can vary up to around $0.7 \mathrm{~K} .{ }^{36}$ We use ternary GUVs as a model system, formed from lipids with a composition chosen to exhibit liquid-disordered/ liquid-ordered phase coexistence with circular lipid domains. ${ }^{20}$ There are three main concepts that we bring together to achieve control over the spontaneous and directed growth of the tubules. First, we control the relative area fractions of liquid ordered and liquid disordered phases, which in turn relates to subtle regulation of vesicle shape and thereby controls internal/external monolayer areas. Second, we include "wedge-shaped" lipids with negative curvature that can stimulate tubule growth from a bilayer, due to a temperature-change induced imbalance in wedge-lipids across the bilayer leaflets. These wedge-lipids enable randomly-oriented tube growth from the vesicle when no thermal gradient is applied, ${ }^{12,31}$ and they stabilise those tubes due to their innate curvature. Third, we implement the recently revealed migration of lipid domains seen in thermal gradients ${ }^{16}$ to reposition lipid domains on the surface of the GUV. Domains migrate towards higher temperatures to reduce their line interface energy closer to mixing. ${ }^{16}$ Following migration of the domains to higher temperatures, tubule growth is induced, giving directed growth on the hot side. This technique could be extended to the directed expulsion of daughter vesicles of known composition, containing moieties segregated into the tube phase.

\section{Experimental}

\subsection{GUV preparation}

Vesicles were formed using electroformation ${ }^{37,38}$ from 1,2dioleoylphosphatidylcholine (DOPC), dipalmitoylphosphatidylcholine (DPPC), dioleoylglycerophosphoethanolamine (DOPE), and cholestanol (chol). Electroformation was carried out at $333 \mathrm{~K}$ at $10 \mathrm{~Hz}$ for 2 hours, followed by $2 \mathrm{~Hz}$ for 1 hour. Lipids were purchased from Avanti Polar Lipids (Alabaster, AL) in chloroform. Cholestanol (Sigma-Aldrich) was used in place of cholesterol to reduce photo-oxidation. A fluorescent lipid, 1,2-dihexadecanoyl-sn-glycero-3-phosphoethanolamine, triethylammonium salt (TX-DHPE), which preferentially partitions into the $\mathrm{L}_{\mathrm{d}}$ phase was included at $0.8 \mathrm{~mol} \%$ (Thermo-Fisher). GUVs were prepared with an inner solution of $197 \mathrm{mM}$ sucrose (SigmaAldrich) and an outer solution of $200 \mathrm{mM}$ glucose (Sigma-Aldrich) so that vesicles sedimented at the bottom surface to facilitate imaging. GUVs were stored at room temperature and imaged within a few hours after electroformation.

GUVs with liquid-disordered $\left(\mathrm{L}_{\mathrm{d}}\right)$ /liquid-ordered $\left(\mathrm{L}_{\mathrm{o}}\right)$ phase coexistence were formed from mixtures of DOPC, DPPC, and chol with a fluorescent probe. Molar ratios of DOPC : DPPC : chol were chosen that formed circular lipid domains of the $\mathrm{L}_{\mathrm{d}}$ phase (or $\mathrm{L}_{\mathrm{o}}$ phase) with a near equal area to that of the background phase. ${ }^{20}$ For circular $\mathrm{L}_{\mathrm{d}}$ domains, we used DOPC : DPPC : chol with a composition of $30: 30: 40 \mathrm{~mol} \%$, and for circular $\mathrm{L}_{\mathrm{o}}$ domains, we used $35: 35: 30 \mathrm{~mol} \%$. Other compositions with the same domain type $\left(\mathrm{L}_{\mathrm{d}}\right.$ or $\mathrm{L}_{\mathrm{o}}$ as the minority phase) behave much in the same manner.

\subsection{Temperature control cell}

The thermal imaging cell (see Fig. 1(a)) was formed from a $200 \mu \mathrm{m}$ thick silicone spacer, with a hole to contain the vesicle suspension, sandwiched between two sapphire windows. Prior to assembling the sample cell, the sapphire windows were boiled in 2\% Hellmanex III commercial surfactant solution and solicated for $15 \mathrm{~min}$, then rinsed thoroughly in MilliQ water and sonicated for a further $15 \mathrm{~min}$ before drying in nitrogen. The windows were placed between metal plates with independently controlled temperatures. Heating and cooling were facilitated by a Peltier system with an assisted circulating water cooling pump to transfer heat away from the device. A central hole cut into the lower plate to grant access for an objective lens enabled imaging of the vesicles in brightfield. Thermocouples were used to measure the temperature of the plates. A linear temperature gradient $(\nabla T)$ was applied vertically with a hotter upper surface, to avoid convection. The temperatures of each plate were held fixed using a custom-built temperature-controller with a feedback loop.

Prior to applying a temperature ramp, we heated GUVs above their transition temperature for $5 \mathrm{~min}$ and cooled them again to ensure lipids within the membrane fully mixed to present a starting point where domains were not already coalesced on the membrane.

During imaging, the cold plate of the thermal-cell was typically held at $278 \mathrm{~K}$ to begin with. For a temperature ramp with $\nabla T \neq 0$, the hot plate was then ramped from $278 \mathrm{~K}$ up to $343 \mathrm{~K}$ in intervals of $1 \mathrm{~K}(\nabla T \neq 0)$ while the cold plate was maintained at $278 \mathrm{~K}$ throughout. The temperature of the cell was allowed to equilibrate for 1 minute between each step. Whereas, for a temperature ramp with $\nabla T=0$, the hot plate was fixed at the same temperature as the cold plate (i.e. $278 \mathrm{~K}$ to start with), and then both were increased together in $1 \mathrm{~K}$ steps, i.e. both to $279 \mathrm{~K}$ and equilibrated for 1 minute, until both plates reached $343 \mathrm{~K}$.

\subsection{Imaging of the GUVs}

Fluorescence imaging of the vesicles was carried out on a Nikon Eclipse Ti-E inverted microscope with a $40 \times$ objective 
a)

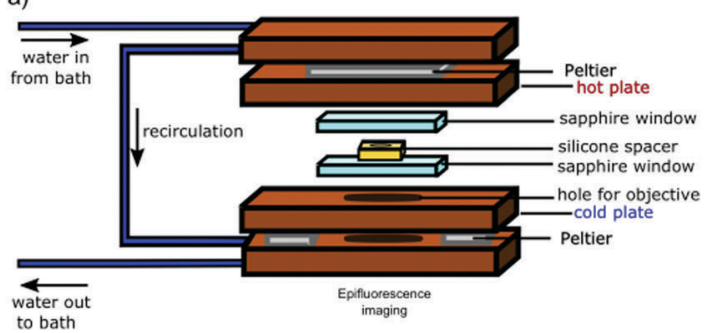

b)

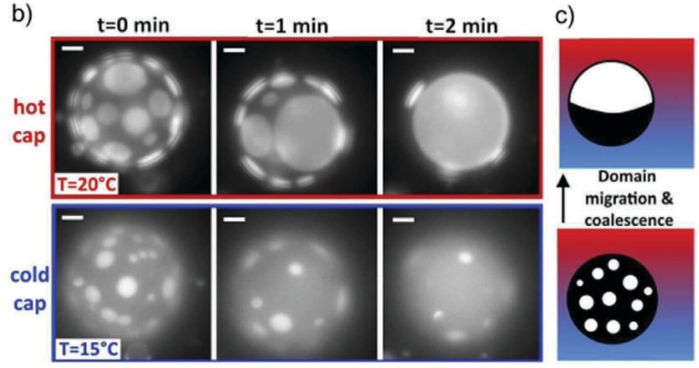

d)

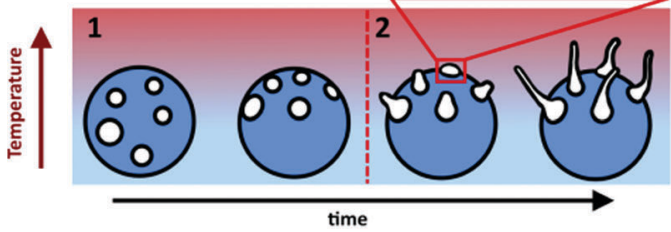

Fig. 1 GUVs are held in a thermal gradient and imaged in epifluorescence. Tubules nucleate from phase domains, which migrate towards the hot cap. (a) The schematic shows the custom-made imaging cell used to impose a thermal gradient: the copper holder dimensions are $54 \mathrm{~mm} \times 86 \mathrm{~mm} \times$ $34 \mathrm{~mm}$. Sapphire windows ensure no lateral convection. The vesicle sample sits inside the hole in the silicone spacer, which ensures a sample thickness of $200 \mu \mathrm{m}$. The range of $\nabla T$ between the hot and cold plates in this work is typically $0-0.33 \mathrm{~K} \mathrm{\mu m}^{-1}$. (b) As in a previous work, ${ }_{1}^{16}$ we observe that the cold cap is depleted of domains (here, $L_{d}$ in DOPC: DPPC: chol $30: 30: 40 \mathrm{~mol} \%$ GUV). Scale bars are $10 \mu \mathrm{m}$. In this time series, the thermal gradient was $\nabla T=0.1 \mathrm{~K} \mathrm{\mu m}^{-1}$ with cap $T$ as indicated. (c) Schematic of domain migration and coalescence from the cold to the hot side, as in a similar system. ${ }^{16}$ (d) Schematic of the directed and position controlled tubule growth observed in this study. Controlled tubule growth results from a two step process. Step 1: circular lipid phase domains of the liquid-disordered phase migrate towards higher temperatures as shown previously. ${ }^{16}$ When present, the wedge-shaped lipid DOPE preferentially partitions into the liquid-disordered phase and is transported with the domains towards higher temperatures. Step 2: tubules grow from domains on the hot side of the vesicle while GUVs are still phase separated when DOPE is present.

lens (Nikon, S Plan Fluor, ELWD 2.8-3.6 mm, NA 0.6) and a camera (Point Grey Research, Grasshopper3 GS3-U3-23S6M). Samples were illuminated with a single-colour LED through a Texas Red filter set (Semrock, exciter FF01-562/40, dichroic FF593-Di02, emitter FF01-624/40). A z-stack was taken across the vesicle and the slices were analysed using custommade MATLAB image analysis routines. Manual contour measurements in MATLAB were used to assess tubule lengths (Fig. 2(b)).
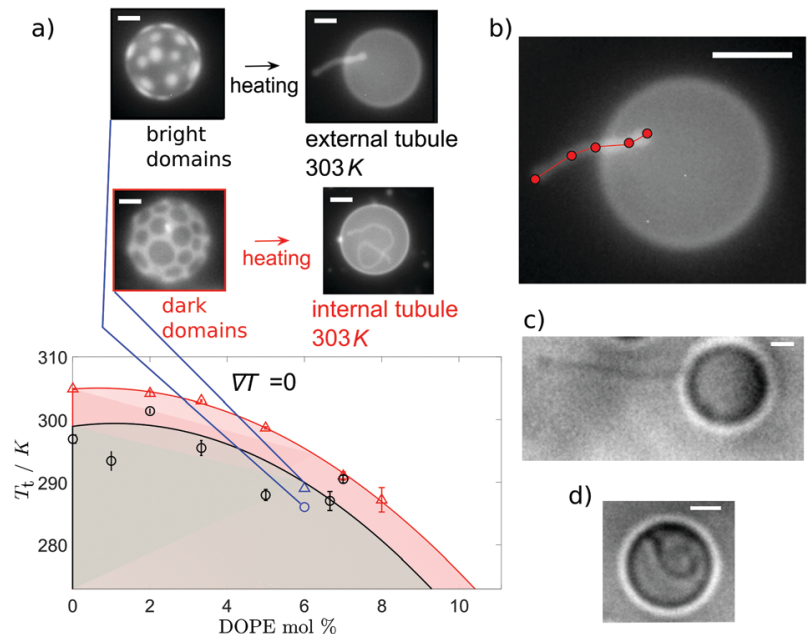

c)

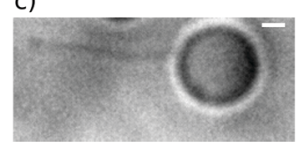

d)

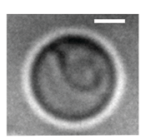

Fig. 2 The direction of tubule growth depends on the composition of the minority phase. Phase domains form below the transition temperature, $T_{\mathrm{t}}$, indicated by the points on (a) for increasing concentrations of the wedge lipid DOPE. Above $T_{\mathrm{t}}$, the lipids are fully mixed. Two lipid compositions are indicated with molar ratios $X$ : DPPC: chol, where $X$ is the mol\% of DOPE + DOPC. These are composition $30: 30: 40 \mathrm{~mol} \%(O)$, which forms GUVs with a bright $L_{d}$ minority phase, and 35:35:30 mol\% $(\triangle)$, which forms GUVs with dark $L_{\circ}$ phase domains. For either composition, $T_{t}$ decreases at higher mol\% of DOPE. Each data point for $T_{\mathrm{t}}$ is the mean of 5-15 GUVs (with standard error bars on the mean). Coloured lines are guides to the eye for each lipid composition series, 30:30:40 mol\% (black) and $35: 35: 30 \mathrm{~mol} \%$ (red). Shaded regions indicate temperatures at which phase domains exist for that composition (30:30:40 mol\% (grey) and $35: 35: 30 \mathrm{~mol} \%$ (pink)). On heating, tubules grow externally from vesicles with $L_{d}$ domains and internally for vesicles with $L_{o}$ domains. Example images of domains and tubulation are shown for $6 \%$ DOPE GUVs with corresponding data in blue ( $O$ or $\triangle$ depending on chol content). (b) Illustration of tubule length measurement, assessed manually by measuring contours in MATLAB. Tubule growth direction depends on the minority phase, independent of the addition of wedge lipid DOPE or the dyed lipid TX-DHPE. Bright field image of GUVs with no wedge lipid or dyed lipid TX-DHPE at $333 \mathrm{~K}$ confirm this for external tubule growth from a GUV with $L_{d}$ domains (c) and internal tubule growth from GUV with $L_{\circ}$ domains (d). All scale bars are $10 \mu \mathrm{m}$.

\section{Results and discussion}

\subsection{Tubule growth in the absence of "wedge-shaped lipids"}

First, we observe the effect of a thermal gradient on GUVs in the absence of "wedge-shaped" lipids, using a mixture of DOPC : DPPC : $\operatorname{chol}(30: 30: 40 \mathrm{~mol} \%)$. When a ternary GUV is quenched below its phase transition temperature, $T_{\mathrm{t}}$, lipid domains form, which diffuse on the membrane and coalesce. When no thermal gradient is applied across the vesicle, coalescence leads to one large domain of the $\mathrm{L}_{\mathrm{o}}$ phase, and one large domain of the $\mathrm{L}_{\mathrm{d}}$ phase with random orientation. In contrast, as we reported recently on a similar system, ${ }^{16}$ by applying a thermal gradient across a GUV with $\mathrm{L}_{\mathrm{o}} / \mathrm{L}_{\mathrm{d}}$, the phase coexistence resulted in lipid domain migration towards the hot side, to minimise the line interface energy (the line interface energy is lower closer to mixing) ${ }^{16}$ (Fig. 1(b and c)). The phase that forms the circular domains migrates towards higher temperatures (the $\mathrm{L}_{\mathrm{d}}$ phase in Fig. 1(b)), and in this manner, the cold hemisphere of a GUV can be depleted of domains. Domains gather onto the hot hemisphere 
(Fig. 1(b and c)), provided the temperature of the hot side remains below the transition temperature, $T_{\mathrm{t}}$. To observe the migration, the temperature was first increased above $T_{\mathrm{t}}$ and held for $5 \mathrm{~min}$ to ensure that the lipids were fully mixed prior to quenching.

When no wedge lipid is included in the membrane, we observe tubule growth from the GUVs occurring upon heating and crossing above the transition temperature, $T_{\mathrm{t}}$. For our choice of ternary compositions, the systems with $\mathrm{L}_{\mathrm{d}}$ circular domains exhibit a transition temperature, $T_{\mathrm{t}}$, slightly lower than the systems with $\mathrm{L}_{\mathrm{o}}$ domains (Fig. 2(a)), and they also grow tubules at lower temperatures. For vesicles that originally have $\mathrm{L}_{\mathrm{d}}$ domains, tubule growth is external to the vesicle. In contrast, for vesicles that originally have $\mathrm{L}_{\mathrm{o}}$ domains, tubules grow into the interior of the vesicle. Examples of each type of growth are shown in Fig. 2(c and d).

The growth of internal tubules from vesicles with $\mathrm{L}_{\mathrm{o}}$ domains plateaus after a given temperature above the initialisation temperature, with normalised tubule lengths comparable for both $\nabla T=0$ and $\nabla T \neq 0$ (Fig. 3(a)). The comparable growth suggests that tubule length depends on the increase in temperature of the vesicle, and not on the thermal gradient. In comparison, the outwardly extending tubules grown from vesicles with $\mathrm{L}_{\mathrm{d}}$ domains exhibit an initial stage of growth followed by a stage of shrinking (Fig. 3(b)). In the first stage of growth, the diameter of the vesicle remains fairly constant, whereas the stage in which the tubule shrinks corresponds to a swelling of the vesicle diameter. Lastly, a new interior tubule sprouts and grows as the vesicle shrinks in size once more. The external-growth/shrink/internal-growth behaviour of these membrane tubules imposes a thermal limit for external tube growth and switch over to internal tube growth, which has potential use as a trigger to introduce a "switch off temperature" after which external tubes will no longer grow to heat but instead go inside the vesicle.

The tubule growth above $T_{\mathrm{t}}$ can be explained by considering the excess area of the bilayer. As the temperature of the membrane increases, the relative area density of each leaflet of the bilayer changes due to the larger change in area of the outer leaflet from thermal expansion, which, for geometric reasons, ${ }^{39}$ leaves an excess area in the outer leaflet (lipid flip-flop might equilibrate this excess over a long period of time). Excess area in the outer leaflet would always promote growth of external tubes on heating. Additionally, as the transition temperature is approached, the lipid compositions of the two phases are brought closer, reducing the ordering of acyl chains in the $\mathrm{L}_{\mathrm{O}}$ phase. The reduction in packing of the lipid tail-groups can increase the excess area of the vesicle (for both leaflets). ${ }^{40}$ So, the average interfacial area of the lipids can increase at higher temperatures, contributing to an increase in the excess area. ${ }^{24}$ For example, a DPPC lipid molecule has an average interfacial area of $47.9 \AA^{2}$ at $293 \mathrm{~K}^{41}$ and $64 \AA^{2}$ at $323 \mathrm{~K}^{41}\left(T_{\mathrm{m}}=314 \mathrm{~K}^{20}\right)$. These factors all couple to result in external tubule growth above $T_{\mathrm{t}}$, to relieve the imbalance in excess membrane area. However, another important aspect is the vesicle shape in the presence of domains, which has been considered in various studies. ${ }^{24,42}$ The disordered phase has a lower bending modulus, and therefore domains of that phase tend to bud out of the majority
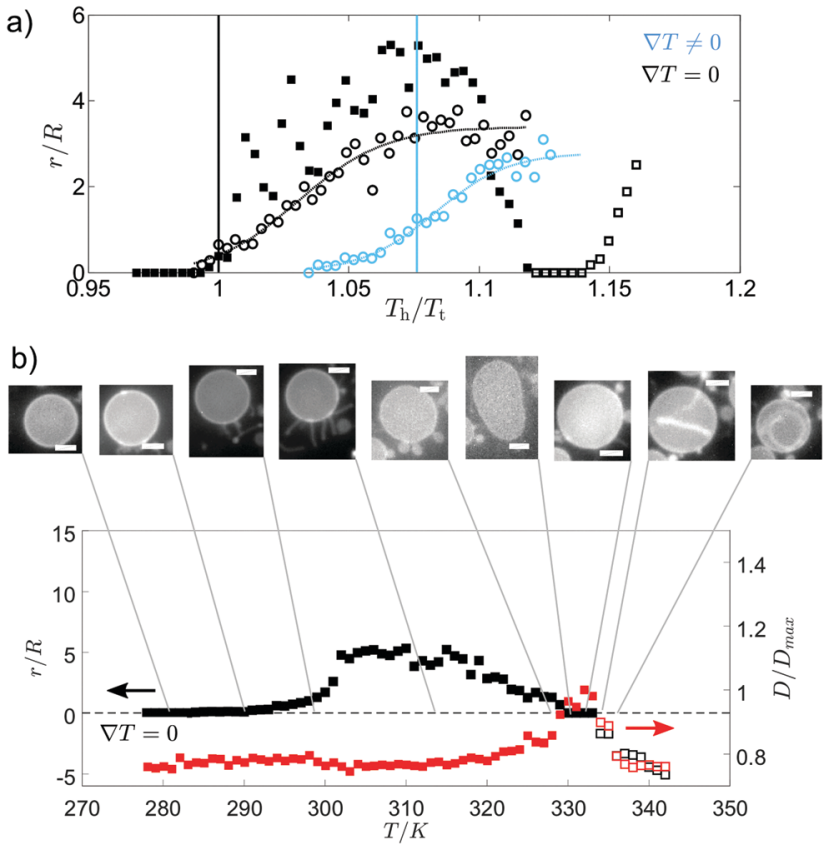

Fig. 3 Tubule growth from vesicles with $L_{d}$ domains begins externally then switches to internal growth. In (a), tubule length is compared for GUVs with $L_{d}\left(\square, 30: 30: 40\right.$ mol\% DOPC : DPPC : chol) or $L_{\circ}$ domains ( $O$, 35 : 35 : 30 mol\% DOPC : DPPC : chol) with and without a thermal gradient. These GUVs do not include wedge lipids. Tubule length, $r$, is shown with respect to GUV radius, $R$, as temperature increases. Tubule growth is shown relative to temperature of the hot plate, $T_{h}$, normalised by the transition temperature, $T_{\mathrm{t}}$. Note that each vesicle composition has a different $T_{\mathrm{t}}$, and so scales differently with temperature. Tubule growth initiated above $T_{\mathrm{t}}$ is fitted by sigmoids (dashed lines). Solid vertical lines indicate the normalised temperature at which the mean temperature of the vesicle is equal to $T_{\mathrm{t}}$ (blue for $\nabla T \neq 0$, black for $\nabla T=0$ ). For GUVs with $L_{d}$ domains, after initial growth, external tubules shrink (closed $\square$ symbols). Then, as the temperature increases further, an internal tubule grows instead (open $\square$ symbols). GUVs with $L_{\circ}$ domains only grow internal tubules (open $\bigcirc$ symbols). (b) The normalised tubule length (black data) and comparative normalised GUV diameter (red data) during heating for a single GUV with $L_{d}$ domains. Arrows indicate the correct axis for each data set. Initial tubule growth is external to the vesicle and occurs while the vesicle diameter is constant. A secondary stage follows where the external tubule shrinks and the vesicle diameter swells. Then, an internal tubule grows (indicated here as negative normalised length) as the vesicle diameter shrinks. Example equatorial images are displayed for each step (scale bars are $10 \mu \mathrm{m}$ ), with the corresponding normalised vesicle diameter and tubule length. No thermal gradient is applied in (b).

ordered phase. In contrast, domains of the ordered phase tend to be flat in the background of a disordered majority phase. As the temperature is increased towards melting, the vesicle approaches a spherical shape (this can be through non-monotonic changes in the diameter ${ }^{24}$ ), and at this point, a vesicle that started with $\mathrm{L}_{\mathrm{d}}$ domains will have reduced overall curvature, whereas a vesicle that started with $\mathrm{L}_{\mathrm{o}}$ domains will have increased curvature. The two conditions lead to opposite excess areas across the bilayer, which can be balanced by forming external and internal tubules, respectively.

When no thermal gradient is applied, tubule growth is initiated at $\sim T_{\mathrm{t}}$, whereas when a thermal gradient is applied, 
tubule growth is initiated when the temperature of the hot side of the cell has far surpassed the transition temperature (Fig. 3(a)). The high $T$ tubulation transition for $\nabla T \neq 0$ could indicate that a large enough proportion of the membrane must reach temperatures above $T_{\mathrm{t}}$ to give a significant enough increase in the excess area for tubule formation. Indeed, the mean normalised temperature at which tubule growth begins in a thermal gradient is close to the point the mean vesicle temperature reaches $T_{\mathrm{t}}$ (Fig. 3(a)). Tubule growth above $T_{\mathrm{t}}$ (when no wedge lipids are present) prevents the use of lipid phase domains to position the tubes, since domains disappear above $T_{\mathrm{t}}$.

Note that the fluorescent dyed lipid, TX-DHPE, has an innate curvature, hence its preference for one phase over the other: as a control, we checked that tubule growth is still initiated at temperatures high above the transition temperature when this dye is not included in the membrane and there are no wedge lipids either (Fig. 2(c and d)).

We also attempted an experiment to test if the tube has a clear lumen accessible to the vesicle interior. Fluorescein dye was included within the vesicle compartment. We could not observe the fluorescein dye signal from the tubule, leaving an open question as to whether the external tubules are bilayer tubes or composed of the outer leaflet only.

\subsection{Phase behaviour and tubule growth in the presence of "wedge-shaped" lipids}

As a further parameter to control tubule growth, we introduced wedge-shaped lipids. DOPE was added in a molar fraction relative to the DOPC content. The molar fraction of lipids that prefer the disordered phase was kept constant (i.e. DOPC + DOPE $=X$ ), as was the fraction of DPPC and chol (e.g. $X$ : DPPC: chol of $30: 30: 40 \mathrm{~mol} \%)$. Addition of DOPE in the membrane stabilises tubule growth through lipid sorting due to the innate curvature of the wedge lipids, ${ }^{43}$ but addition of even small molar fractions of DOPE significantly lowers the transition temperature (Fig. 2(a)). Therefore, a molar fraction of 66\% DOPE was used in GUV formation to ensure stable tubules while enabling the use of large enough thermal gradients for domain migration.

When no thermal gradient is applied, the DOPE:DOPC: DPPC:chol vesicles with $\mathrm{L}_{\mathrm{d}}$ domains deform from spherical and then grow tubules external to the vesicle as $T_{\mathrm{t}}$ is exceeded. As the temperature is increased above $T_{\mathrm{t}}$, there is a local increase in excess area, predominantly of the $\mathrm{L}_{\mathrm{o}}$ background phase, which makes the vesicle floppy (see Fig. S1a, ESI $\dagger$ ) and enables randomly orientated tubule formation. Similar to the vesicles without the wedge lipid, external tubules grew and then shrank, before becoming internal tubules instead. Vesicles with $\mathrm{L}_{\mathrm{o}}$ circular domains containing the wedge lipid grow internal tubules, also initiated after $T_{\mathrm{t}}$ has been exceeded for $\nabla T=0$ (Fig. S1b, ESI $\dagger$ ). At higher temperatures, tubule growth is enhanced independent of the phase of the domains, which is explained by the increase in excess area with temperature.

For GUVs with $\mathrm{L}_{\mathrm{d}}$ domains, the DOPE is concentrated within these disordered domains, favouring negative curvature of the inner leaflet and leading to the formation of external tubes. If instead, the GUVs have $\mathrm{L}_{\mathrm{o}}$ domains, then the DOPE is more spread out in the background phase. In this case, the increased excess area of the GUV is mostly local to the domains and lowers the internal pressure, and an invaginated tubule forms to relax the negative pressure.

\subsection{Directed tubule growth in a thermal gradient}

Having determined the phase behaviour of the DOPE:DOPC: DPPC:chol vesicles and established the growth of tubules controlled by temperature, we explored the potential for directed tubule growth in a thermal gradient, starting with GUVs exhibiting $\mathrm{L}_{\mathrm{d}}$ circular domains. By enabling domain migration onto the hot side of the vesicle at temperatures well below $T_{\mathrm{t}}$ before tubule growth begins (Fig. 4(a)), then enhancing domain migration and tubule growth by increasing the thermal gradient (Fig. 4(b)), tubules can be directed from the $\mathrm{L}_{\mathrm{d}}$ domains outward from the vesicle surface. As the $\mathrm{L}_{\mathrm{d}}$ domains are confined to the hot side of the vesicle, so are the tubules. Therefore, material confinement within domains and subsequent redistribution through domain migration provide a route for the directed growth of protuberances. This method combines control of domain patterning and curvature using a thermal trigger.

If tubule growth occurs before domains have fully migrated to the hot cap, then tubules can grow from both caps of the

a)
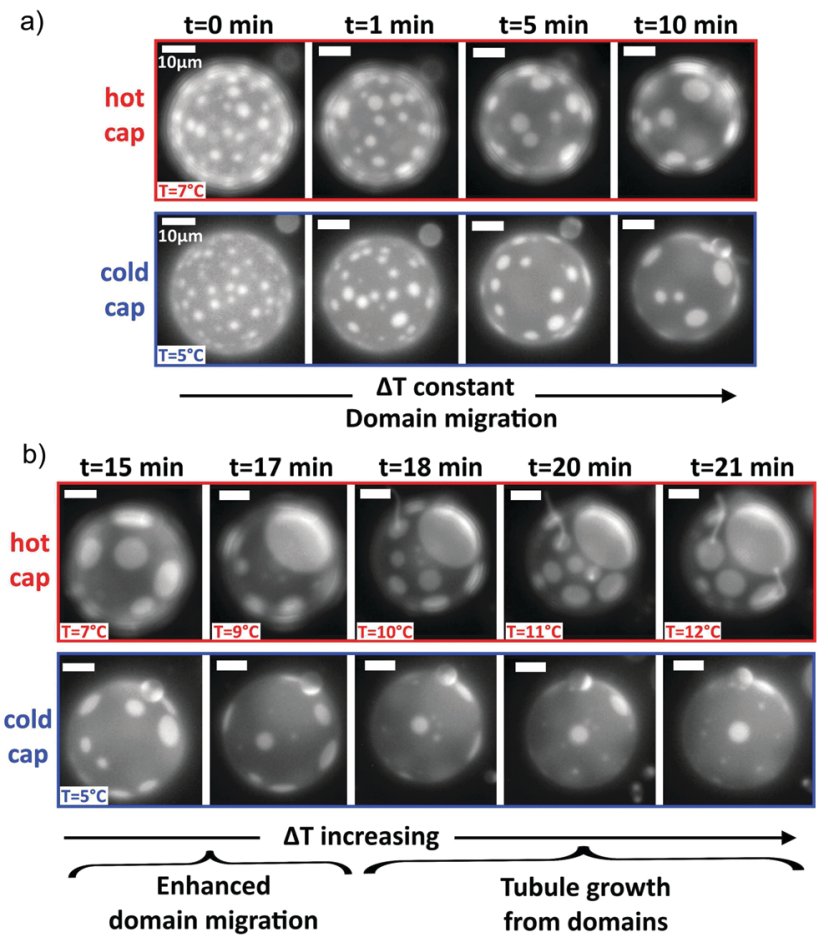

Fig. 4 Domain migration coupled with inclusion of wedge lipids in the membrane enables directed tubule growth external to the GUV from $L_{d}$ circular domains. (a) Domains on a GUV of DOPE: DOPC:DPPC: chol $(6: 21.5: 27.5: 45 \mathrm{~mol} \%)$ migrate when $\nabla T \neq 0$ towards the hot cap and coalesce. The cold cap is depleted of the $L_{d}$ domains, which are rich in DOPE. (b) Increasing $\nabla T$ enhances domain depletion. As the temperature of the hot side, $T_{h}$, is further increased, tubules grow from $L_{d}$ domains on the hot cap. The temperature of each cap is indicated. The thermal gradient was initially $0.05 \mathrm{~K} \mathrm{\mu m}^{-1}$ and later increased to $0.15 \mathrm{~K} \mathrm{\mu m}^{-1}$. Scale bars are $10 \mu \mathrm{m}$. 
vesicle (e.g. Fig. S2, ESI $\dagger$ ). This situation can occur if the temperature across the vesicle becomes high enough to cause tubule growth before the domains have fully migrated onto the hot cap. Similarly, if the temperature is increased above $T_{\mathrm{t}}$ such that extended tubules grow, which remain intact upon quenching below $T_{\mathrm{t}}$, then those tubules do not necessarily associate with $\mathrm{L}_{\mathrm{d}}$ domains and move around the vesicle (Fig. S2, ESI $\dagger$ ), so these tubules can remain on the cold side. To control tubule position, it is therefore important to ensure that tubule growth is only initiated after domain formation and their migration to the hot side.

By changing the composition of the GUV, $\mathrm{L}_{\mathrm{o}}$ circular domains are formed on $\mathrm{L}_{\mathrm{d}}$ background phase, which still migrate around from the cold cap to the hot cap when a thermal gradient is applied (Fig. S3a, ESI $\dagger$ ). As the temperature approaches $T_{\mathrm{t}}$, tubule growth begins, but it is interior to the vesicle (Fig. S3b, ESI $\dagger$ ), persisting above $T_{\mathrm{t}}$ to lengthen the tubules (Fig. S3c, ESI $\dagger$ ). In this case, the temperature gradient does not provide useful directed growth of the tubules from the hot or cold cap, rather the tubules coil inside the vesicle as they extend.

Independent of which phase forms the circular domains, tubule growth in a thermal gradient occurs before passing through the mixing transition temperature for GUVs containing DOPE (Fig. 5). This contrasts with the observations for $\nabla T=0$, where tubule growth was only initiated from the vesicle after $T_{\mathrm{t}}$ was exceeded (Fig. 5), and it is likely in part due to the stabilisation effect of wedge lipids on tubule growth. At a uniform temperature, GUVs containing the wedge lipid grow tubules above the transition temperature with similar growth behaviour to membranes without the wedge lipid (Fig. 5).

For directed tubule growth via domain migration, tubules must form below $T_{\mathrm{t}}$, which is only possible when wedge lipids are included in the membrane and a thermal gradient is applied. The thermal gradient concentrating DOPE by the domain migration mechanism could therefore be a key factor in tubule growth below $T_{\mathrm{t}}$ and in the shrink/growth behaviour.

For vesicles in a thermal gradient with bright $\mathrm{L}_{\mathrm{d}}$ domains, the tubule growth external to the GUV attains longer tubule lengths (compared to the vesicle radius) than vesicles with dark $\mathrm{L}_{\mathrm{o}}$ domains that grow internal tubes (Fig. S4a, ESI $\dagger$ ). The longer growth relates to the larger proportion of the $\mathrm{L}_{\mathrm{o}}$ phase in GUVs with bright domains that is responsible for the largest increase in excess area above $T_{\mathrm{t}}$. For all GUVs, the tubule length initially grows with increasing temperature of the hot side of the vesicle. Tuning the temperature gradient therefore enables control over the length of the tube. In addition, normalised tubule lengths were longer for GUVs containing the wedge lipid when a thermal gradient was applied compared to growth at a uniform temperature.

For GUVs including DOPE, there is then a stage where the tube length shrinks after passing above $T_{\mathrm{t}}$ (Fig. 5). In the case of GUVs with $\mathrm{L}_{\mathrm{o}}$ domains, the internal tubules then re-grow following this regime of shrinking. For GUVs with $\mathrm{L}_{\mathrm{d}}$ domains, shrinking occurs further above $T_{\mathrm{t}}$ and external tubules shrink before internal tubules grow. Tubule shrinkage could occur on passing above $T_{\mathrm{t}}$ due to phase mixing. Below $T_{\mathrm{t}}$, the DOPE is partitioned between each phase with higher concentrations

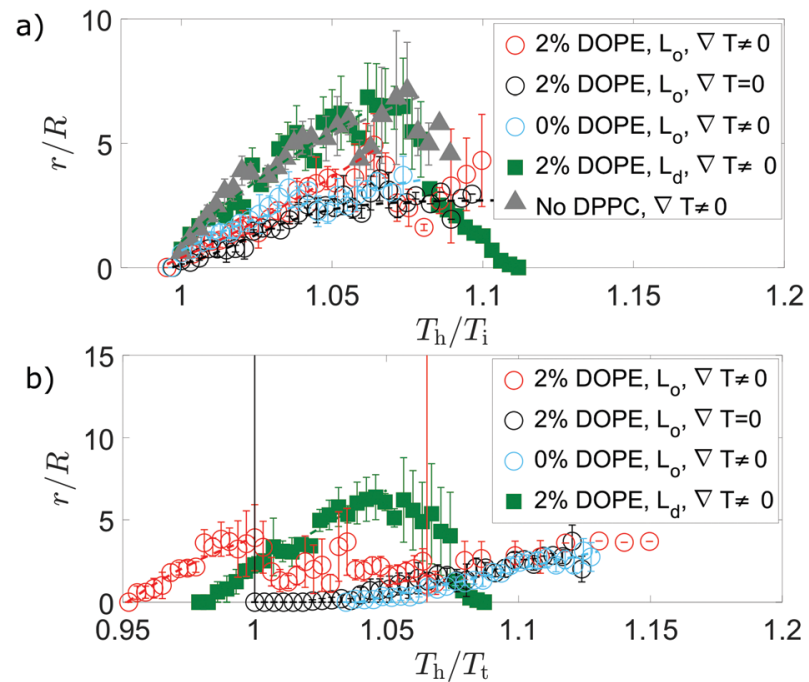

Fig. 5 When there is a temperature gradient and DOPE is included in the membrane, tubules grow below the transition temperature. Tubule growth for different lipid compositions shows initial sigmoidal growth (dashed lines) for internal tubules (dark domains $\bigcirc$ ), external tubules (bright domains $\square$ ), or no domains at all $(\triangle)$. In (a), temperature is normalised by $T_{i}$, the temperature at which tubules initially grow; the same data (matching markers) are instead normalised by the transition temperature, $T_{\mathrm{t}}$, in (b). Data points are mean values for different GUVs, each of which may have a different $T_{\mathrm{i}}$ or $T_{\mathrm{t}}$ value. In a thermal gradient, GUVs with composition DOPE : DOPC: DPPC: chol (2:33: $35: 30$ mol\%) grow internal tubules below $T_{\mathrm{t}}$ that shrink above $T_{\mathrm{t}}$ before re-growth. Solid vertical lines indicate the normalised temperature at which the mean temperature of the vesicle is equal to $T_{\mathrm{t}}$ for $2 \%$ DOPE compositions. When no thermal gradient is applied, tubules grow only above $T_{\mathrm{t}}$, where the lipids are mixed. For GUVs with $L_{d}$ domains (bright fluorescence), there is no re-growth of external tubules after shrinking within the temperature range explored. Vesicles with the same composition ratio as GUVs with $L_{\circ}$ domains, but DOPC replacing all DPPC (i.e. DOPE : DOPC: DPPC : chol 2:68:0:30 mol\%), do not have phase domains and grow external tubules with similar growth to the tubes as in the GUVs with $L_{d}$ domains. Error bars are standard errors on the mean of two or more measurements on different GUVs, each with usually a single tubule.

in the $\mathrm{L}_{\mathrm{d}}$ phase (from which tubules grow for either GUV composition). The DOPE stabilises tubule growth, which is initiated from the line interface between domains in phase separated GUVs. On mixing, the DOPE spreads out in the membrane, locally reducing the concentration of wedge lipid in regions that were previously the $\mathrm{L}_{\mathrm{d}}$ phase, including the tubules. The tubules are then less stable and shrink.

To determine whether the existence of phase domains has a significant contribution to selecting whether tubules grow inward/outward and if they shrink after an initial growth period, vesicles were made with the same composition ratio as GUVs forming $\mathrm{L}_{\mathrm{O}}$ domains but with DOPC replacing all DPPC (i.e. DOPE:DOPC:DPPC: chol 2:68:0:30 mol\%). These GUVs do not have phase domains and they grow external tubules, despite having the same concentration of wedge lipid and cholesterol as GUVs with $\mathrm{L}_{\mathrm{o}}$ domains that have internal tubes. The growth of these external tubes is very similar to growth from GUVs with bright $\mathrm{L}_{\mathrm{d}}$ domains (Fig. 5). This tubule growth plateaus without 
shrinkage (Fig. 5), suggesting phase domains and subsequent phase mixing are necessary for tubule shrinkage. Additionally, the length of tubules grown either internally or externally correlates positively with an increase in GUV radius, suggesting a strong link with the membrane area (Fig. S4a, ESI $\dagger$ ).

\section{Conclusions}

Directed growth of tubules was achieved from giant unilamellar vesicles with liquid-liquid phase coexistence using a thermal gradient and lipids with negative curvature. Below the phase transition temperature, circular lipid domains formed on the surface of the GUVs, with the domain phase dependent on the vesicle composition. The thermal gradient induced the motion of the circular domains, causing migration towards higher temperatures. The migration occurred due to the lower line interface energy at higher temperatures (i.e. the force generated by the difference in line interface energy across a domain) and depleted circular domains from the cold cap, crowding domains onto the hot cap. When negative curvature (wedge-shaped) lipids were included in the vesicles, tubule growth occurred even below the phase transition temperature. This is interesting because the degree of curvature in GUVs, in itself, is not enough to lead to significant partitioning; ${ }^{43}$ we propose that there is a geometrical effect linked to heat induced expansion that leads to leaflet imbalance.

The phase transition temperature was significantly decreased on addition of more wedge-shaped lipids. For vesicles with circular domains formed from the liquid-ordered phase, tubule growth was interior to the vesicle. However, for vesicles with circular domains formed from the liquid-disordered phase, tubules grew outwards from the vesicle's surface and to longer lengths (compared to the GUV radius). In the latter case, tubule growth was induced following the migration of domains to the hot side of the vesicle by raising the temperature of the hot cap. The result was the directed growth of tubules from the hot side of the vesicle. Hence, material concentration of wedge-shaped lipids within domains and the subsequent redistribution of those domains through thermally-induced migration provide a route for the directed growth of protuberances, offering significant potential to advance avenues of synthetic biology.

\section{Conflicts of interest}

There are no conflicts to declare.

\section{Acknowledgements}

This work was supported by EPSRC (grant number EP/J017566/1). ET was supported by the Wellcome Trust through a Junior Interdisciplinary Research Fellowship. LDM acknowledges support from the Leverhulme Trust and the Isaac Newton Trust through an Early Career Fellowship (ECF-2015-494) and from the Royal Society through a University Research Fellowship (UF160152).

\section{References}

1 E. Gutlederer, T. Gruhn and R. Lipowsky, Soft Matter, 2009, 5, 3303-3311.

2 U. Seifert and R. Lipowsky, Handbook of Biological Physics, Elsevier Science B.V., 1995, vol. 1.

3 N. Sciaky, J. Presley, C. Smith, K. Zaal, N. Cole, J. Moreira, M. Terasaki, E. Siggia and J. Lippincott-Schwartz, J. Cell Biol., 1997, 139, 1137-1155.

4 C. Lee and L. Chen, Cell, 1988, 54, 37-46.

5 M. McNiven and H. Thompson, Science, 2006, 313, 1591-1594.

6 R. Lipowsky, Faraday Discuss., 2013, 161, 305-331.

7 J. Martínez-Menárguez, ISRN Cell Biol., 2013, 2013, 126731.

8 E. Talbot, J. Kotar, L. Parolini, L. Di Michele and P. Cicuta, Nat. Commun., 2017, 8, 1-8.

9 M. Kozlov, H. McMahon and L. Chernomordik, Trends Biochem. Sci., 2010, 35, 699-706.

10 A. Callan-Jones, B. Sorre and P. Bassereau, Cold Spring Harbor Perspect. Biol., 2011, 3, a004648.

11 L. Feriani, L. Cristofolini and P. Cicuta, Chem. Phys. Lipids, 2015, 185, 78-87.

12 J. Zimmerberg and M. Kozlov, Nat. Rev. Mol. Cell Biol., 2016, 7, 9-19.

13 J. Hu, Y. Shibata, C. Voss, T. Shemesh, Z. Li, M. Coughlin, M. Kozlov, T. Rapoport and W. Prinz, Science, 2008, 319, 1247-1250.

14 Z. Shi and T. Baumgart, Nat. Commun., 2015, 6, 1-8.

15 B. Kelly and D. Owen, Curr. Opin. Cell Biol., 2011, 23, 404-412.

16 E. Talbot, L. Parolini, J. Kotar, L. Di Michele and P. Cicuta, Proc. Natl. Acad. Sci. U. S. A., 2017, 114, 846-851.

17 V. Noireaux and A. Libchaber, Proc. Natl. Acad. Sci. U. S. A., 2004, 101, 17669-17674.

18 Y. Elani, R. Law and O. Ces, Nat. Commun., 2014, 5, 1-5.

19 C. Schmitt, A. Lippert, N. Bonakdar, V. Sandoghdar and L. Voll, Front. Bioeng. Technol., 2016, 4, 1-12.

20 S. Veatch and S. Keller, Biophys. J., 2003, 85, 3074-3083.

21 P. Cicuta, S. Keller and S. Veatch, J. Phys. Chem. B, 2007, 111, 3328-3331.

22 A. Honerkamp-Smith, P. Cicuta, M. Collins, S. Veatch, M. den Nijs, M. Schick and S. Keller, Biophys. J., 2008, 95, 236-246.

23 S. Veatch, K. Gawrisch and S. Keller, Biophys. J., 2006, 90, 4428-4436.

24 Y.-Z. Yoon, J. Hale, P. Petrov and P. Cicuta, J. Phys.: Condens. Matter, 2010, 22, 062101.

25 A. Karlsson, R. Karlsson, M. Karlsson, A.-S. Cans, A. Strömberg, F. Ryttsén and O. Orwar, Nature, 2001, 409, 150-152.

26 G. Koster, A. Cacciuto, I. Derényi, D. Frenkel and M. Dogterom, Phys. Rev. Lett., 2005, 94, 068101.

27 R. Dasgupta and R. Dimova, J. Phys. D: Appl. Phys., 2014, 47, 282001.

28 O. Rossier, D. Cuvelier, N. Borghi, P. Puech, I. Derényi, A. Buguin, P. Nassoy and F. Brochard-Wyart, Langmuir, 2003, 19, 575-584.

29 G. Koster, M. Vanduijn, B. Hofs and M. Dogterom, Proc. Natl. Acad. Sci. U. S. A., 2003, 100, 15583-15588. 
30 J. Stachowiak, C. Hayden and D. Sasaki, Proc. Natl. Acad. Sci. U. S. A., 2010, 107, 7781-7786.

31 Y. Sakuma, T. Taniguchi, T. Kawakatsu and M. Imai, Biophys. J., 2013, 105, 2074-2081.

32 Y.-C. Tan, A. Shen, Y. Li, E. Elson and L. Ma, Lab Chip, 2008, 8, 339-345.

33 Y. Li, R. Lipowsky and R. Dimova, Proc. Natl. Acad. Sci. U. S. A., 2011, 108, 4731-4736.

34 Y. Liu, J. Agudo-Canalejo, A. Grafmuller, R. Dimova and R. Lipowsky, ACS Nano, 2016, 10, 463-474.

35 J.-B. Fournier, N. Khalifat, N. Puff and M. Angelova, Phys. Rev. Lett., 2009, 102, 018102.

36 K. Okabe, N. Inada, C. Gota, Y. Harada, T. Funatsu and S. Uchiyama, Nat. Commun., 2012, 3, 1-9.
37 M. Angelova and D. Dimitrov, Faraday Discuss. Chem. Soc., 1989, 81, 303-311.

38 M. Angelova, S. Soléau, P. Méléard, J.-F. Faucon and P. Bothorel, Prog. Colloid Polym. Sci., 1992, 89, 127-131.

39 S. Svetina, B. Zeks, R. Waugh and R. Raphael, Eur. Biophys. J., 1998, 27, 197-209.

40 D. Chapman, Q. Rev. Biophys., 1975, 8, 185-235.

41 J. Nagle and S. Tristram-Nagle, Biochim. Biophys. Acta, 2000, 1469, 159-195.

42 S. Semrau, T. Idema, T. Schmidt and C. Storm, Biophys. J., 2009, 96, 4906-4915.

43 M. M. Kamal, D. Mills, M. Grzybek and J. Howard, Proc. Natl. Acad. Sci. U. S. A., 2009, 106, 22245-22250. 